\title{
SPECTRAL CLASSIFICATION OF B AND A STARS FROM DATA OF S2/S68 EXPERIMENT
}

\author{
A. CUCCHIARO \\ Institut d'Astrophysique, Université de Liège, Belgium \\ and \\ M. JASCHEK, C. JASCHEK, \\ Observatoire de Strasbourg, France \\ and \\ D. MACAU-HERCOT* \\ Institut d'Astrophysique, Université de Liège, Belgium
}

\begin{abstract}
The S2/S68 experiment on the satellite TD1 A has supplied, in the wavelength region of $1350 \AA$ to $2550 \AA$, a very large number of spectra of early stars.

A statistical study, as well as a general analysis of these spectra, has been carried out in order to establish criteria relative to the spectral region envisaged and independent of any previous study in the visible. On the basis of these criteria a system of classification has been outlined.

In a first stage, the spectra of stars visually classed from B0 to A5 have been considered. From four sets of spectrophotometric criteria a two-dimensional ultraviolet classification has been derived.
\end{abstract}

The purpose of this note is to present a tentative ultraviolet spectral classification of B and A stars from the data of the S2/S68 experiment.

Since the classification in view is a lengthy task, it has been necessary to proceed by successive stages. The first approach was to consider the early B stars. The spectra of the early B stars are characterized by 3 features situated at $\lambda \lambda 1400 \AA, 1550 \AA$ and $1620 \AA$ and have been attributed mainly to C IV and Fe III ( $\lambda$ 1550) by Peytremann (1975) and Swings et al. (1974), Si IV ( $\lambda$ 1400) and Fe II ( $\lambda$ 1620) by Swings et al. (1974).

If the following ratios:

$$
\frac{R_{1}}{R_{2}}=\frac{\text { absolute flux at the bottom of the } 1 \text { st feature }}{\text { absolute flux at the bottom of the } 2 \text { nd feature }}
$$

and

$$
\frac{R_{3}}{R_{2}}=\frac{\text { absolute flux at the bottom of the } 3 \text { rd feature }}{\text { absolute flux at the bottom of the } 2 \text { nd feature }}
$$

are considered and the two values put in a graph, a separation of the sample spectra becomes possible. In fact, it appears that 8 typical regions of the plane can be defined.

Now if the points of region are considered as belonging to one and the same family of points and if for each region a graph, where $R_{1} / R_{2}$ is plotted in function of $r_{1}+r_{2}$, is drawn, three new regions of points can be defined. As an example, the Figures $1 \mathrm{a}$ and $1 \mathrm{~b}$

* Chercheur qualifié au F.N.R.S.

B. Hauck and P. C. Keenan (eds), Abundance Effects in Classification, 177-180. All Rights Reserved. Copyright $\odot 1976$ by the IAU. 


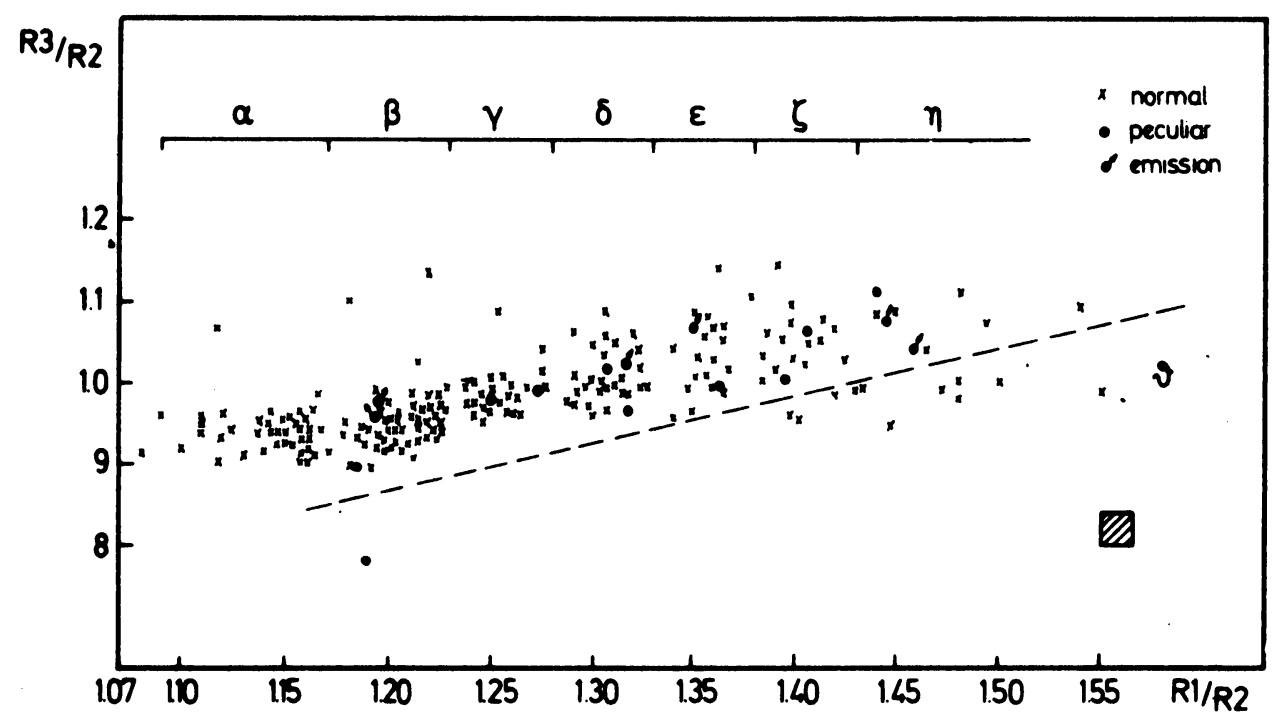

Fig. 1a. First separation for the early B stars sample in 8 families of points.

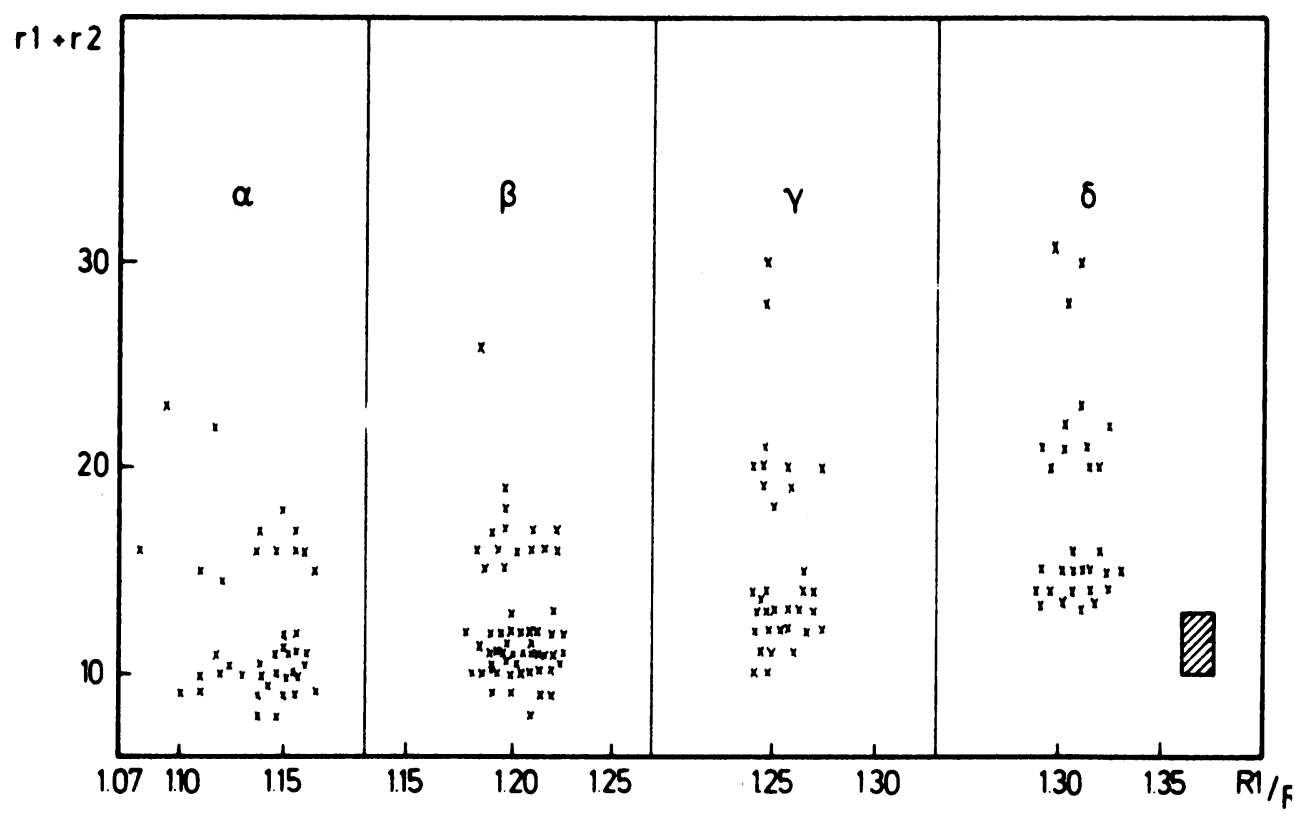

Fig. 1b. For each $\alpha, \beta, \gamma, \delta$ families of points, a second separation into three sub-groups.

show the two-dimensional separation for the early B stars. $r_{1}$ and $r_{2}$ are the intrinsic depth of the 1st and 2nd feature defined from a pseudo continuum (Malaise et al., 1974).

The correlation between this ultraviolet classification and the Yerkes classification both with regard to temperature and to luminosity class gives the first 8 families of points 
$(\alpha, \beta, \gamma, \delta, \epsilon, \zeta, \eta, \theta)$ corresponding in general to B5, B3, B2.5, B2, B1.5, B1, B0.5, BO MK type stars.

For each family the three other regions correspond in general to:

- main-sequence stars

- giant stars

- supergiant stars.

The second stage was to consider the late B stars.

A main characteristic of the ultraviolet spectrum of a late B star is the $\lambda \lambda 2440 \AA$ feature attributed to Fe II by Swings et al. (1974).

If the next quantity:

$$
A=1-\left(\frac{F_{r}}{F_{c}}\right)_{2400},
$$

where $F_{r 2400}$ is the absolute flux at the bottom of the feature and $F_{c 2400}$ is the absolute flux calculated for the pseudo continuum at $2400 \AA$, is plotted as a function of $m_{1400}-m_{v}$, a separation of the sample into four families of points is also possible.

Now, as for the early B stars, if $m_{1400}-m_{2730}$ is given vs the $A$ value for each family of points, a second subdivision appears.

Correlation with the MK spectral types shows that the first 4 families correspond to B6, B7, B8, B9 and B9.5 stars.

The sub-groups for each family correspond to

- main-sequence stars

- giant stars

- supergiant stars.

The last step was to analyze the early A stars. As for the late B stars, a main characteristic of the A ultraviolet spectrum is the $\lambda \lambda 2400 \AA$ feature but attributed for the early A stars to Ni II and Fe II by Gros et al. (1973).

If the following ratios:

$$
\frac{F_{2400}}{F_{2300}}
$$

and

$$
\frac{F_{2400}}{F_{2730}}
$$

are taken into account, one can show that, as for the two preceding stages, five regions of the plane can be well defined and the correlation with the MK spectral type shows that the $\nu, \xi, o, \pi$ and $\rho$ families correspond in general to A0, A1, A2, A3 and A5 stars. 


\section{Conclusion}

The preceding considerations show that the ultraviolet spectra of B and early A stars can be classified my means of ultraviolet criteria exclusively.

For more details a substantial paper will be published later.

\section{References}

Gros, M., Sacotte, D., Praderie, F., and Bonnet, R. M.: 1973, Astron. Astrophys. $27,167$.

Malaise, D., Beeckmans, F., and Jamar, C.: 1974, European Regional Meeting in Astronomy, Trieste, (in press).

Peytremann, E.: 1975, Astron. Astrophys. (in press).

Swings, J. P., Jamar, C., and Vreux, J. M.: 1973, Astron. Astrophys. 29, 207.

\section{DISCUSSION}

Gerbaldi: Did you observe some Am stars and what did you notice?

Cucchiaro: Yes, we have observed Am stars; It appears that some of them escaped from our classification scheme and others agreed with it. But the number of Am stars of our sample is very low until now and any conclusions can be outlined on an eventual separation between Am and normal A stars.

McCarthy: What do you observe as peculiar features in the location on your plots of the Be stars as compared to the non-emission B stars?

Cucchiaro: The Be stars as compared to the non-emission B stars don't present striking characteristics in this wavelength region. Their place in the present classification, except for a few of them, corresponds to what is known from visual spectroscopy.

Garrison: Perhaps I missed an important point, but I don't understand why the results shown in your diagrams are quantized according to MK types and luminosity classes. Is it that God believes in MK classification?

Cucchiaro: We have asked ourselves such a question and until now no satisfactory answer has been found. Perhaps the number of spectra is too small, yet I believe that to give a real answer we can analyse all the physical processes which correspond to our criteria. 